\title{
Clinical characteristics of different histologic types of breast cancer
}

\author{
CI Li',', DJ Uribe' and JR Daling' \\ 'Division of Public Health Sciences, Fred Hutchinson Cancer Research Center, I 100 Fairview Ave. N., M4-C308, PO Box 19024, Seattle, \\ WA 98109-1024, USA
}

Breast cancer is a heterogeneous disease, though little is known about some of its rarer forms, including certain histologic types. Using Surveillance, Epidemiology, and End Results Program data on 135 I57 invasive breast cancer cases diagnosed from 1992 to 200 I, relationships between nine histologic types of breast cancer and various tumour characteristics were assessed. Among women aged 50-89 years at diagnosis, lobular and ductal/lobular carcinoma cases were more likely to be diagnosed with stage III/IV, $\geqslant 5.0 \mathrm{~cm}$, and node-positive tumours compared to ductal carcinoma cases. Mucinous, comedo, tubular, and medullary carcinomas were less likely to present at an advanced stage. Lobular, ductal/lobular, mucinous, tubular, and papillary carcinomas were less likely, and comedo, medullary, and inflammatory carcinomas were more likely to be oestrogen receptor (ER) negative/progesterone receptor (PR) negative and high grade (notably, 68.2\% of medullary carcinomas were ER-/PR- vs 19.3\% of ductal carcinomas). In general, similar differences were observed among women diagnosed at age 30-49 years. Inflammatory carcinomas are associated with more aggressive tumour phenotypes, and mucinous, tubular, and papillary tumours are associated with less aggressive phenotypes. The histologic types of breast cancer studied here differ greatly in their clinical presentations, and the differences in their hormone receptor profiles and grades point to their likely different aetiologies.

British Journal of Cancer (2005) 93, I046- 1052. doi:I0.1038/sj.bjc.6602787 www.bjcancer.com

Published online 20 September 2005

(C) 2005 Cancer Research UK

Keywords: breast carcinoma; histology; SEER; stage; oestrogen receptor

\begin{abstract}
Although breast cancer remains the most commonly diagnosed cancer among women in the United States (US) and worldwide, it is a heterogeneous disease. Breast cancer can be categorized in several ways, including based on its clinical features, its expression of tumour markers, and its histologic type. The two most common histologic types of invasive breast cancer are ductal and lobular carcinomas, accounting for approximately 75 and $15 \%$ of all cases in the US, respectively (Li et al, 2003a). Interest in lobular carcinoma in particular has recently been piqued by data indicating that incidence rates of lobular carcinoma are increasing more rapidly than are rates of ductal carcinoma in the US. Specifically, lobular rates have increased by 65\% from 1987 to 1999, while rates of ductal carcinoma have increased only by $3 \%$ (Li et al, 2003a). Studies also suggest that lobular carcinomas are more likely than ductal carcinomas to be hormone receptor positive (Arpino et al, 2004; Korhonen et al, 2004). This difference may partly explain why seven studies have consistently observed that combined oestrogen and progestin postmenopausal hormone use is more strongly related to lobular carcinoma risk than to ductal carcinoma risk (Li et al, 2000, 2003b; Chen et al, 2002; Newcomb et al, 2002; Daling et al, 2003; Newcomer et al, 2003).
\end{abstract}

While several studies have now examined the clinical, pathologic, and epidemiologic differences between ductal and lobular carcinomas, much less is known about the rarer histologic types of

*Correspondence: Dr Cl Li; E-mail: cili@fhcrc.org

Received 27 June 2005; revised 5 August 2005; accepted 15 August 2005; published online 20 September 2005 breast cancer, including mucinous, tubular, comedo, inflammatory, medullary, and papillary carcinomas, which together account for about $10 \%$ of all cases. The purpose of this study is to characterise how rare histologic types of breast cancer differ in their stage, size, lymph node status, oestrogen receptor (ER)/PR status, and grade, utilizing data from 11 population-based tumour registries that participate in the Surveillance, Epidemiology, and End Results (SEER) Program. Evaluations of these differences are important for furthering our understanding of the nature of these tumours and may provide insight into the aetiologies and clinical features of different types of breast cancer.

\section{MATERIALS AND METHODS}

Women diagnosed with a first primary invasive breast cancer at 30-89 years of age between January 1992 and December 2001 were identified through 11 population-based cancer registries in the US that participate in the National Cancer Institute's SEER Program. This age range was chosen because the vast majority of breast cancers diagnosed in the US occur among women in this age range ( $>98 \%$ based on the SEER data used here). Also, different aetiologic factors likely influence breast cancer occurrence among women younger than 30 or older than 90 years of age, but since too few women are diagnosed in these age ranges we could not assess them separately. The SEER registries that were used include those serving the states of Connecticut, Hawaii, Iowa, New Mexico, and Utah, and the urban areas surrounding Atlanta, GA; Detroit, MI; Los Angeles, CA; San Francisco-Oakland, CA; San Jose-Monterey, $\mathrm{CA}$; and Seattle, WA. The standard for ascertainment of cases of 
cancer in the SEER registries is 98\% (Surveillance Epidemiology and End Results, National Cancer Institute, 2005a). Individual patient medical records are the source of SEER data on patient and tumour characteristics. In general, the populations covered by SEER are representative of the whole US with regard to socioeconomic status and education level, though they include higher proportions of people living in urban areas and who are foreign born (Surveillance Epidemiology and End Results, National Cancer Institute, 2005b). Further operational details and methods used by the SEER Program are provided elsewhere (Young Jr et al, 1981).

In all, 199721 women 30-89 years of age whose invasive breast cancer diagnosis was their first primary cancer diagnosis of any type were eligible for this study. Also, 539 women whose cancers were either not microscopically confirmed or were diagnosed only at autopsy were excluded. Women were grouped into the following nine histologic categories based on the ICD-O-3 code assigned to their tumours using an approach that has been previously published (Li et al, 2003c): ductal (defined using ICD-O code 8500), lobular (8520), ductal/lobular (8522), mucinous (8480), tubular (8211), comedocarcinoma (8501), inflammatory (8530), medullary (8510), and papillary (8050 and 8503). In addition, SEER's Extent of Disease (EOD) codes were used to identify additional inflammatory cases, since SEER EOD code 70 is used to define 'inflammatory carcinoma, including diffuse (beyond that directly overlying the tumour) dermal lymphatic permeation or infiltration'. Hence, cases with a SEER EOD code of 70 were defined as inflammatory, regardless of the ICD-O-3 code they were assigned. The 10167 women with other ICD-O codes, representing $5.1 \%$ of eligible subjects, were excluded from our analysis, leaving a total of 189015 women. This group included women with diagnoses such as Paget's disease, because there were too few cases with these diagnoses to evaluate them separately in our analysis. In addition, we excluded 1116 subjects $(5.9 \%$ of the total potentially eligible subjects) because they had an unknown or missing race/ ethnicity. Since we were particularly interested in differences in ER and PR status by histology, we also excluded 52742 cases $(28.1 \%$ of the total potentially eligible subjects) because data on their ER and/or PR status were unknown. After these exclusions, 135157 eligible women remained, including 102463 ductal, 11275 lobular, 9636 ductal/lobular, 3248 mucinous, 2222 comedo, 2095 inflammatory, 1983 tubular, 1617 medullary, and 618 papillary carcinoma cases.

In addition to histologic type, SEER registries also collect data on other tumour characteristics including AJCC stage at diagnosis, tumour size, lymph node status, ER and PR status, and tumour grade. Associations between the histologic type of breast cancer and each of these tumour characteristics were estimated using polytomous logistic regression (Begg and Gray, 1984), and based on likelihood ratio testing that compared models that included and excluded the tumour characteristic of interest, each of these tumour characteristics was statistically significant overall $(P<0.00001$ for each characteristic). We stratified our main analyses by age at diagnosis, evaluating the 100028 women diagnosed at age 50-89 years and the 35129 women diagnosed at age 30-49 years separately, because breast cancer risk factors, outcomes, and tumour characteristics differ by age, and particularly by menopausal status, and thus age is likely to be an important modifier of the associations assessed here (Bernstein, 1998). In the absence of information on menstrual history, 50 years of age has been shown to be a reasonable proxy for distinguishing postmenopausal from premenopausal women (Morabia and Flandre, 1992). Using Stata SE for Windows (Stata Corporation, College Station, TX, USA) statistical software, odds ratios (OR) and 95\% confidence intervals (CI) were calculated. In all analyses, ductal carcinoma cases served as the reference histology group. All analyses were adjusted for age at diagnosis (continuous), year of diagnosis (continuous), SEER registry (categorical), and race/ ethnicity (categorical). In addition, our analyses of stage, tumour size, and lymph node status were also adjusted for ER/PR status, our analyses of ER status were also adjusted for PR status, our analyses of PR status were also adjusted for ER status, and our analyses of tumour grade were also adjusted for stage and ER/PR status because each were hypothesized as potential confounders. Age at diagnosis (categorized as 30-49 years vs 50-89 years of age) was found to be an effect modifier of each of the relationships we assessed based on likelihood ratio testing, since $P$-values for interaction were all $<0.05$. In fact, all $P$-values for interaction were $<0.00001$, except those for tumour size $(P=0.0014)$ and nodal status $(P=0.0149)$.

Certain associations were not evaluated because the histopathologic definition of certain histologic types of breast cancer is dependent on the presence of particular clinical or pathologic features. Specifically, inflammatory carcinoma is defined by tumour emboli in dermal lymphatic vessels, and since $>99 \%$ of inflammatory cases included in this study were stage III or stage IV and $98 \%$ were $\geqslant 5.0 \mathrm{~cm}$ we did not evaluate stage and tumour size differences among inflammatory cases. Also, since tubular carcinomas are by definition well differentiated, and $99 \%$ of tubular cases in this study were grade 1 or 2 , we did not evaluate tumour grade differences among tubular cases. Finally, since medullary carcinomas are characteristically poorly differentiated, and $90 \%$ of medullary cases in this study were grade 3 or 4 , we did not evaluate tumour grade differences among medullary cases.

\section{RESULTS}

Of the nine histologic types assessed, mucinous and papillary carcinoma cases had the oldest mean ages at diagnosis (65.8 and 65.7 years, respectively) and medullary carcinoma cases had the youngest mean age at diagnosis (52.8 years) (Table 1$)$. While the number of ductal, lobular, ductal/lobular, mucinous, tubular, and inflammatory carcinoma cases increased over time, the number of comedo and medullary carcinoma cases declined and the number of papillary carcinoma cases held fairly constant. Variations in the racial/ethnic compositions of cases were also observed by histologic type.

Among women diagnosed with breast cancer at age 50-89 years of age, women with lobular, ductal/lobular, and inflammatory carcinoma were statistically more likely to be diagnosed with stage III/IV disease, while mucinous, tubular, comedo, and medullary carcinoma cases were less likely, compared to women with ductal carcinoma (Table 2). With respect to tumour size, women with lobular, ductal/lobular, and papillary carcinomas were more likely to be diagnosed with tumours that were $5.0 \mathrm{~cm}$ or larger $(13.0,7.5$, and $9.5 \%$ of these women had tumours $\geqslant 5.0 \mathrm{~cm}$, respectively) compared to ductal carcinoma cases (5.6\%), while tubular carcinoma cases were less likely $(0.8 \%)$. Lobular, ductal/lobular, and inflammatory carcinoma cases also were more likely to be diagnosed with node-positive disease. Alternatively, mucinous, comedo, tubular, medullary, and papillary cases all were less likely to be diagnosed with node-positive disease. With respect to hormone receptor status, lobular, ductal/lobular, mucinous, tubular, and papillary carcinoma cases were $0.2-0.4$-fold less likely to be diagnosed with $\mathrm{ER}-/ \mathrm{PR}$ - tumours, while comedo, medullary, and inflammatory cases were $1.7-11.6$-fold more likely to be diagnosed with $\mathrm{ER}-/ \mathrm{PR}$ - tumours compared to ductal carcinoma cases. Finally, compared to ductal carcinoma cases, women with mucinous and papillary carcinomas tended to have lower-grade tumours, while comedo and inflammatory carcinoma cases tended to have higher-grade tumours.

Similar to the older women, among women diagnosed with breast cancer at age 30-49 years of age, those with lobular, and ductal/lobular carcinomas were more likely to be diagnosed with stage III/IV disease, while mucinous, comedo, tubular, and 
Table I Demographic characteristics of 139310 women diagnosed with nine different histologic types of breast cancer

\begin{tabular}{|c|c|c|c|c|c|c|c|c|c|c|c|c|c|c|c|c|c|c|}
\hline & \multicolumn{2}{|c|}{$\begin{array}{c}\text { Ductal } \\
(n=102463)\end{array}$} & \multicolumn{2}{|c|}{$\begin{array}{c}\begin{array}{c}\text { Lobular } \\
(n=11275)\end{array} \\
\end{array}$} & \multicolumn{2}{|c|}{$\begin{array}{c}\text { Ductal/ } \\
\text { lobular } \\
(n=9636)\end{array}$} & \multicolumn{2}{|c|}{$\begin{array}{l}\text { Mucinous } \\
(n=3248)\end{array}$} & \multicolumn{2}{|c|}{$\begin{array}{c}\text { Comedo } \\
(n=2222)\end{array}$} & \multicolumn{2}{|c|}{$\begin{array}{c}\text { Inflammatory } \\
(n=2095)\end{array}$} & \multicolumn{2}{|c|}{$\begin{array}{c}\text { Tubular } \\
(n=1983)\end{array}$} & \multicolumn{2}{|c|}{$\begin{array}{l}\text { Medullary } \\
(n=1617)\end{array}$} & \multicolumn{2}{|c|}{$\begin{array}{l}\text { Papillary } \\
(n=618)\end{array}$} \\
\hline \multicolumn{19}{|l|}{ Age at diagnosis } \\
\hline $30-39$ & 6911 & 7 & 225 & 2 & 428 & 4 & 119 & 4 & 234 & 11 & 190 & 9 & 42 & 2 & 249 & 15 & 21 & 3 \\
\hline $40-49$ & 20754 & 20 & 1663 & 15 & 1849 & 19 & 399 & 12 & 616 & 28 & 518 & 25 & 344 & 17 & 497 & 31 & 70 & 11 \\
\hline $70-79$ & 19932 & 20 & 2820 & 25 & 1932 & 20 & 1005 & 31 & 287 & 13 & 308 & 15 & 397 & 20 & 153 & 10 & 177 & 29 \\
\hline $80-89$ & 7860 & 8 & $124 \mid$ & 11 & 687 & 7 & 508 & 16 & 82 & 4 & $|4|$ & 7 & 109 & 6 & 47 & 3 & 101 & 16 \\
\hline Mean \pm s.d. & \multicolumn{2}{|c|}{$59.5 \pm 13.6$} & \multicolumn{2}{|c|}{$63.4 \pm 12.7$} & \multicolumn{2}{|c|}{$60.1 \pm 12.9$} & \multicolumn{2}{|c|}{$65.8 \pm 13.5$} & \multicolumn{2}{|c|}{$55.3 \pm 13.1$} & \multicolumn{2}{|c|}{$60.7 \pm 11.7$} & \multicolumn{2}{|c|}{$57.0 \pm 13.8$} & \multicolumn{2}{|c|}{$52.8 \pm 12.9$} & \multicolumn{2}{|c|}{$65.7 \pm 13.2$} \\
\hline \multicolumn{19}{|l|}{ Diagnosis year } \\
\hline $1992-1993$ & 17157 & 17 & |79| & 16 & 1209 & 13 & 524 & 16 & 800 & 36 & 338 & 16 & 239 & 12 & 424 & 26 & 118 & 19 \\
\hline $1994-1995$ & 18445 & 18 & 1975 & 18 & 1377 & 14 & 571 & 18 & 553 & 25 & 338 & 16 & 331 & 17 & 342 & 21 & 133 & 22 \\
\hline Non-Hispanic white & 78748 & 77 & 9605 & 85 & 7908 & 82 & 2467 & 76 & 1568 & 71 & 1518 & 73 & 1726 & 87 & 1004 & 62 & 434 & 70 \\
\hline Black & 7927 & 8 & 555 & 5 & 563 & 6 & 199 & 6 & 246 & 11 & 253 & 12 & 71 & 4 & 287 & 18 & 69 & 11 \\
\hline Asian/Pacific Islander & 8745 & 9 & 484 & 4 & 534 & 6 & 363 & 11 & 238 & 11 & 124 & 6 & 93 & 5 & 127 & 8 & 68 & 11 \\
\hline Hispanic white & 6693 & 7 & 614 & 5 & 603 & 6 & 212 & 7 & 161 & 7 & 185 & 9 & 91 & 5 & 188 & 12 & 44 & 7 \\
\hline American Indian & 350 & 0.3 & 17 & 0.2 & 28 & 0.3 & 7 & 0.2 & 9 & 0.4 & 15 & 0.7 & 2 & 0.1 & 11 & 0.7 & 3 & 0.5 \\
\hline
\end{tabular}

s.d. $=$ standard deviation

medullary carcinoma cases were less likely, compared to women with ductal carcinoma (Table 3). With respect to tumour size women with lobular and ductal/lobular carcinomas were more likely to be diagnosed with tumours that were $5.0 \mathrm{~cm}$ or larger (18.1 and $12.1 \%$ of these women had tumours $\geqslant 5.0 \mathrm{~cm}$, respectively) compared to ductal carcinoma cases $(8.6 \%)$, while tubular and medullary carcinoma cases were less likely (1.0 and $5.6 \%$, respectively). Lobular, ductal/lobular, and inflammatory carcinoma cases also were more likely to be diagnosed with nodepositive disease, and mucinous, comedo, tubular, and medullary cases were less likely, compared to ductal cases. In addition, lobular, ductal/lobular, mucinous, tubular, and papillary carcinoma cases were $0.2-0.5$-fold less likely to be diagnosed with ER-/ PR - tumours, while comedo, medullary, and inflammatory cases were 1.9-15.3-fold more likely to be diagnosed with ER-/PRtumours compared to ductal carcinoma cases. Finally, compared to ductal carcinoma cases, women with lobular and mucinous carcinomas tended to have lower-grade tumours, while comedo and inflammatory carcinoma cases tended to have higher-grade tumours.

\section{DISCUSSION}

Before interpreting the results of this study, it is important to acknowledge its limitations. First, the histologic categorizations used were based on diagnoses made by multiple pathologists in multiple institutions, and diagnostic criteria may vary somewhat by both individual pathologists and institutions, resulting in a certain degree of misclassification error. Studies evaluating the concordance between histologic classifications of breast tumours ascertained by SEER registries and those made through a centralized pathology review are needed to quantify the magnitude of this misclassification, as none have been reported in the literature. However, the misclassification of histologic type present in the SEER data is likely to be nondifferential, and as a result it may obscure differences but not lead to the identification of spurious differences. Also encouraging is that the proportions of cases in each histopathologic category were relatively similar across the 11 registries included in this study. One exception was that $10.9 \%$ of cases diagnosed in Los Angeles were ductallobular, while only $2.9 \%$ diagnosed in Hawaii were ductal-lobular. However, across the remaining nine registries, the proportions of cases that were ductal-lobular were relatively similar. Another concern is that we lacked information regarding certain potential confounders, including hormonal, reproductive, anthropometric, and lifestyle factors, that may be associated with both different histologic types of breast cancer and with the different clinical and pathologic tumour characteristics we evaluated. However, the strengths of this study are that it is large and population-based. Thus, it provides information on the tumour characteristics of different histologic types of breast cancer, some of which are quite rare, that are being observed in the general population.

Differences between various histologic types of breast cancer have been noted in prior studies. Two studies have explored the age distribution of different histologic types of breast cancer and have observed some striking differences. The first study by Stalsberg and Thomas (1993) reported that, while the relative frequency of ductal carcinoma is essentially constant by age, the frequencies of papillary and mucinous carcinomas tend to increase with age, the frequencies of medullary and inflammatory carcinomas tend to decrease with age, and the frequencies of lobular and tubular carcinomas increase until age 50, after which they remain fairly constant. A recent update of age-specific rates by histologic type observed three different age-rate patterns (Anderson et al, 2004). Specifically, rates of ductal, lobular, and tubular carcinomas were shown to rise sharply until age 50 and then rise more slowly, rates of papillary and mucinous carcinomas to rise steadily with age, and rates of medullary and inflammatory carcinoma to increase until age 50, after which they did not continue to rise. While this study also evaluated age-specific 
Table 2 Relationship between breast cancer histology and tumour stage, ER/PR status, and grade among women diagnosed at 50-89 years of age ${ }^{a}$

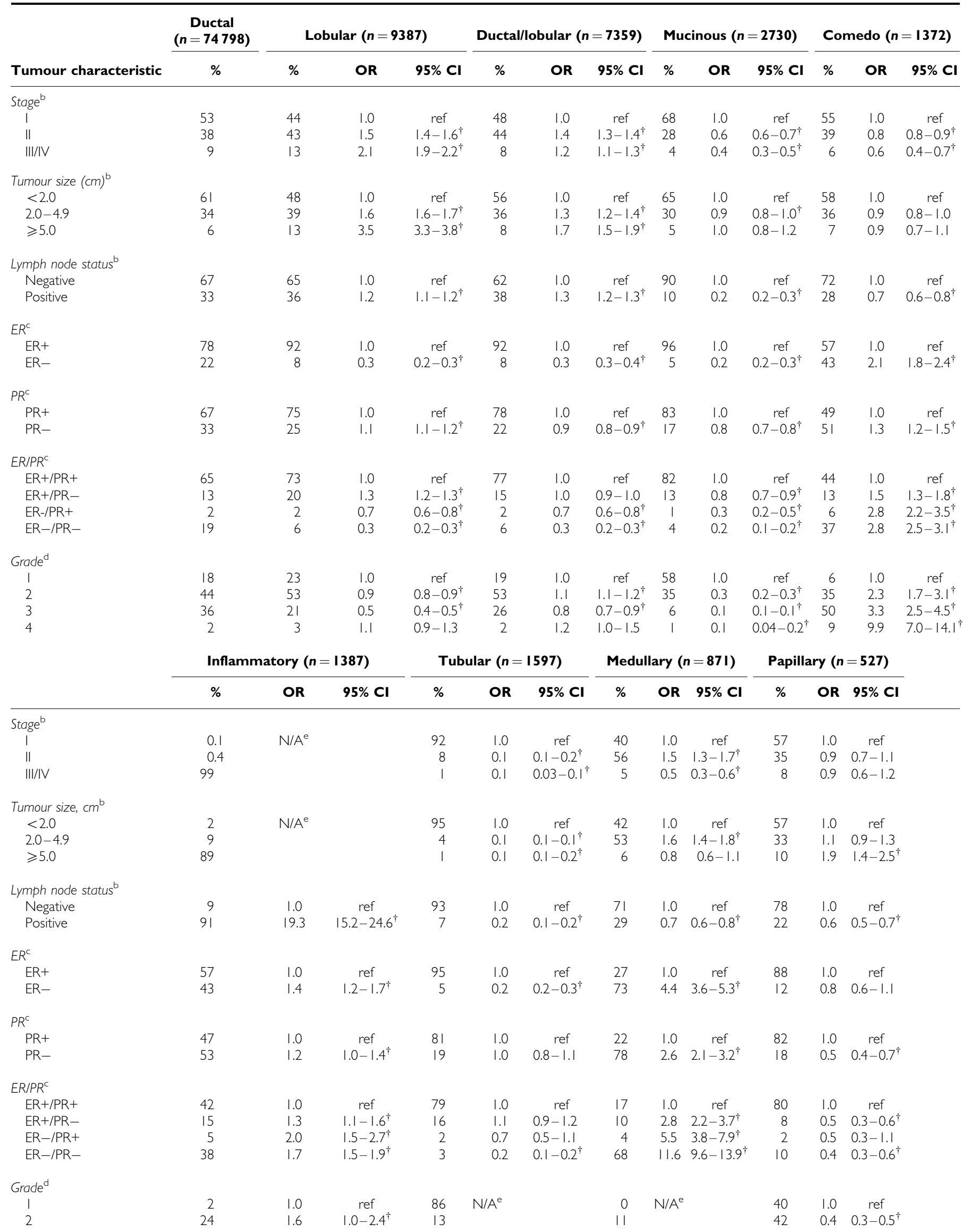


Table 2 (Continued)

\begin{tabular}{|c|c|c|c|c|c|c|c|c|c|c|c|c|}
\hline Tumour characteristic & \multicolumn{3}{|c|}{ Inflammatory $(n=1387)$} & \multicolumn{3}{|c|}{ Tubular $(n=1597)$} & \multicolumn{3}{|c|}{ Medullary $(n=87 I)$} & \multicolumn{3}{|c|}{ Papillary $(n=527)$} \\
\hline $\begin{array}{l}3 \\
4\end{array}$ & $\begin{array}{l}68 \\
67\end{array}$ & $\begin{array}{l}2.4 \\
3.0\end{array}$ & $\begin{array}{l}1.6-3.6^{\dagger} \\
1.9-4.8^{\dagger}\end{array}$ & $\begin{array}{l}1 \\
0\end{array}$ & & & $\begin{array}{l}78 \\
11\end{array}$ & & & $\begin{array}{r}17 \\
2\end{array}$ & $\begin{array}{l}0.2 \\
0.4\end{array}$ & $\begin{array}{l}0.1-0.3^{\prime} \\
0.2-0.8^{i}\end{array}$ \\
\hline
\end{tabular}

$\mathrm{OR}=$ odds ratio, $\mathrm{Cl}=$ confidence interval, $\mathrm{ER}=$ oestrogen receptor, $\mathrm{PR}=$ progesterone receptor, $\mathrm{N} / \mathrm{A}=$ not applicable. ${ }^{\dagger} \mathrm{P}<0.05 .{ }^{\mathrm{a}}$ The reference histologic type for all analyses was ductal carcinoma. ${ }^{b}$ ORs are adjusted for age and year at diagnosis, cancer registry, race/ethnicity, and ER/PR status. Data on tumour size missing for 7 I 0 ductal, I 30 lobular, 34 ductal/lobular, 10 mucinous, eight comedo, one tubular, three medullary, 94 inflammatory, and three papillary carcinomas. Data on lymph node status missing for 805 I ductal, 100I lobular, 565 ductal/lobular, 464 mucinous, 136 comedo, 336 tubular, 43 medullary, 587 inflammatory, and 99 papillary carcinomas. ${ }^{\circ}$ ORs are adjusted for age and year at diagnosis, cancer registry, race/ethnicity, and stage. In addition, ORs for ER status are adjusted for PR status, and ORs for PR status are adjusted for ER status. ${ }^{\mathrm{d} O R s}$ are adjusted for age and year at diagnosis, cancer registry, race/ethnicity, stage, and ER/PR status. Data on grade missing for 5732 ductal, 4 I 49 lobular, 984 ductal/lobular, 98 I mucinous, 367 comedo, 270 tubular, 32 I medullary, 223 inflammatory, and I 40 papillary carcinomas. ${ }^{~}$ These ORs were not calculated because almost all inflammatory carcinomas are stage III or IV and $>5.0 \mathrm{~cm}$ in size, almost all tubular carcinomas are well differentiated, and almost all medullary carcinomas are poorly differentiated.

Table 3 Relationship between breast cancer histology and tumour stage, hormone receptor status, and grade among women diagnosed at $30-49$ years of age $\mathrm{e}^{\mathrm{a}}$

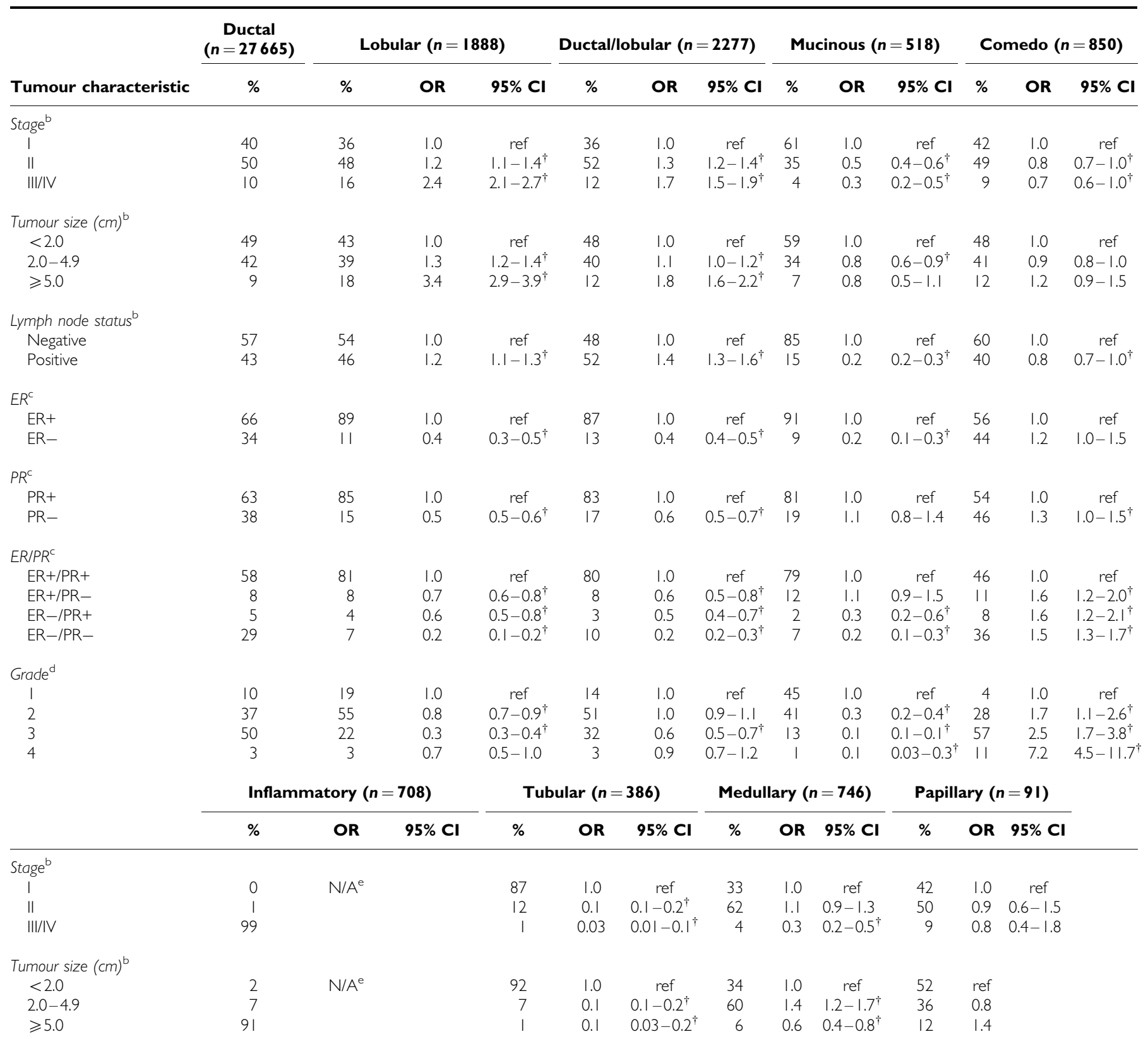


Table 3 (Continued)

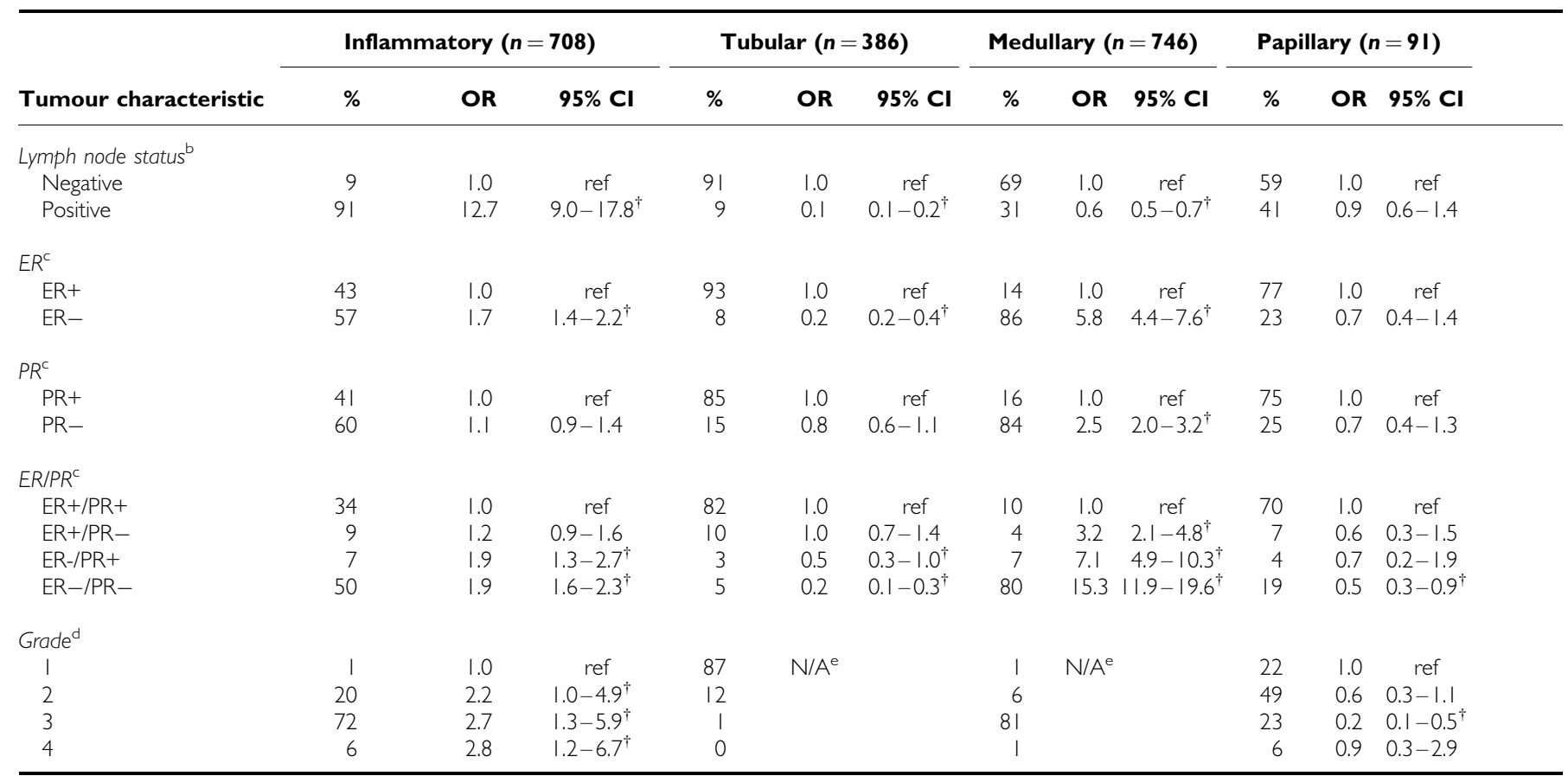

$\mathrm{OR}=$ odds ratio, $\mathrm{Cl}=$ confidence interval, $\mathrm{ER}=$ oestrogen receptor, $\mathrm{PR}=$ progesterone receptor, $\mathrm{N} / \mathrm{A}=$ not applicable. ${ }^{\dagger} \mathrm{P}<0.05 .{ }^{\mathrm{a}}$ The reference histologic type for all analyses was ductal carcinoma. ${ }^{b}$ ORs are adjusted for age and year at diagnosis, cancer registry, race/ethnicity, and ER/PR status. Data on tumour size missing for 209 ductal, 24 lobular, 17 ductal/lobular, three mucinous, five comedo, 36 inflammatory, and one papillary carcinomas. Data on lymph node status missing for I 242 ductal, I I 8 lobular, 87 ductal/lobular, 28 mucinous, 54 comedo, 26 tubular, 17 medullary, 304 inflammatory, and six papillary carcinomas. 'ORs are adjusted for age and year at diagnosis, cancer registry, race/ethnicity, and stage. In addition, ORs for ER status are adjusted for PR status, and ORs for PR status are adjusted for ER status. ${ }^{\mathrm{d}}$ ORs are adjusted for age and year at diagnosis, cancer registry, race/ethnicity, stage, and ER/PR status. Data on grade missing for I 732 ductal, 794 lobular, 289 ductal/lobular, 198 mucinous, 215 comedo, 75 tubular, 25 I medullary, I02 inflammatory, and 22 papillary carcinomas. ${ }^{e}$ These ORs were not calculated because almost all inflammatory carcinomas are stage III or IV and greater than $5.0 \mathrm{~cm}$ in size, almost all tubular carcinomas are well differentiated, and almost all medullary carcinomas are poorly differentiated.

incidence rates by histologic type for ER + and ER - tumours separately, it did not directly compare the distributions of ER status, or other clinical or tumour characteristics by histologic type.

In addition, risk of mortality has been observed to vary by histologic type as women with inflammatory breast cancers have an increased risk of mortality compared to women with other histologic types of breast cancer (Stierer et al, 1993; Anderson et al, 2003), while women with lobular, mucinous, comedo, tubular, medullary, and papillary carcinomas have lower risks of mortality compared to women with ductal carcinomas (Li et al, 2003c). However, few previous studies have evaluated differences in clinical and pathologic tumour characteristics by histologic type. With respect to ER/PR status, lobular, ductal/lobular, and mucinous carcinomas have been shown to be more likely to be $\mathrm{ER}+/ \mathrm{PR}+$ compared to ductal carcinomas, similar to what we observed here (Desai et al, 2000; Arpino et al, 2004; Korhonen et al, 2004; Mathieu et al, 2004). However, studies have not evaluated ER/PR status in rarer histologic types of breast cancer, or evaluated differences in stage, tumour size, lymph node status, or grade by histologic type.

Here we observed several differences in these characteristics by histologic type, which only varied somewhat by age at diagnosis. The primary differences seen between our groups of largely postmenopausal women $v s$ largely premenopausal women were differences in magnitudes rather than directions of risk. For example, among comedo carcinoma cases, the observed elevated risks of ER-/PR - tumours compared to ductal cases were more pronounced among the older women $(\mathrm{OR}=2.8)$ than among the younger women $(\mathrm{OR}=1.5)$. Exceptions to this were that lobular carcinomas diagnosed among younger women were less likely to be $\mathrm{PR}$ - compared to ductal cases, though this difference was not seen among the older women. In addition, older, but not younger, women with comedo carcinoma had an elevated risk of ERtumours. Finally, the higher risk of tumours $\geqslant 5.0 \mathrm{~cm}$ and the lower risk of node-positive tumours seen among older women with papillary carcinomas were not observed among younger women with papillary tumours. However, our ability to detect differences among papillary cases 30-49 years of age was limited by a relatively small sample size $(n=91)$.

Despite these differences, among both age groups of women, mucinous, tubular, and papillary carcinomas generally had less aggressive phenotypes compared to ductal carcinoma cases, as they were each less likely to present at an advanced stage, to be node positive, to be hormone receptor negative, and to have a high grade. These features may explain why women with these tumours have relatively low risks of mortality (Stierer et al, 1993; Li et al, 2003c). In contrast, inflammatory carcinomas appear to have the most aggressive phenotype of any of the histologic types evaluated as these tumours were more likely to be node positive, to be hormone receptor negative, and to have a high grade. These findings are consistent with the poorer survival rates that women with these tumours experience, particularly for those with ERinflammatory carcinoma (Anderson et al, 2003; Li et al, 2003c). Lobular, ductal/lobular, and comedo and medullary carcinomas had mixed phenotypes. Lobular and ductal/lobular tumours tended to be diagnosed at a more advanced stage and to be both $>5.0 \mathrm{~cm}$ and node positive, but they were also much more likely to be hormone receptor positive. In contrast, comedo and medullary carcinomas were less likely to have an advanced stage at diagnosis and to be node positive, but more likely to be hormone receptor negative and to have a high grade. Interestingly, these 
characteristics appear to translate into all of these tumours having lower risks of mortality compared to ductal carcinoma cases, as we have reported previously (Li et al, 2003c).

Beyond differences in their histopathologic appearances, the results of this study suggest that different histologic types of breast cancer also differ substantially in their clinical and tumour characteristics. Interestingly, while inflammatory carcinomas are more likely to be characterized by poor clinical and pathologic tumour characteristics that do translate into poorer survival rates; lobular, ductal/lobular, comedo, and medullary carcinomas are characterized by a mix of tumour characteristics that are associated with both better and poorer prognoses, though previous data indicate that women with these tumours have lower risks of mortality than do women with ductal carcinomas (Li et al, 2003c). Thus, the prognostic importance and utility of the clinical and tumour characteristics evaluated here varies by histologic type. The differences in hormone receptor status and grade that we observed by histologic type may reflect the different aetiologies of these tumours. Further, the differences in stage, tumour size, and

\section{REFERENCES}

Anderson WF, Chu KC, Chang S (2003) Inflammatory breast carcinoma and noninflammatory locally advanced breast carcinoma: distinct clinicopathologic entities? J Clin Oncol 21: 2254-2259

Anderson WF, Chu KC, Chang S, Sherman ME (2004) Comparison of agespecific incidence rate patterns for different histopathologic types of breast carcinoma. Cancer Epidemiol Biomarkers Prev 13: 1128-1135

Arpino G, Bardou VJ, Clark GM, Elledge RM (2004) Infiltrating lobular carcinoma of the breast: tumor characteristics and clinical outcome. Breast Cancer Res 6: R149-R156

Begg CB, Gray R (1984) Calculation of polychotomous logistic regression parameters using individualized regressions. Biometrika 71: 11-18

Bernstein L (1998) The epidemiology of breast cancer. Women Cancer 1S: $7-13$

Chen CL, Weiss NS, Newcomb P, Barlow W, White E (2002) Hormone replacement therapy in relation to breast cancer. JAMA 287: 734-741

Daling JR, Malone KE, Doody DR, Voigt LF, Bernstein L, Marchbanks PA, Coates RJ, Norman SA, Weiss LK, Ursin G, Burkman RT, Deapen D, Folger SG, McDonald JA, Simon MS, Strom BL, Spirtas R (2003) Association of regimens of hormone replacement therapy to prognostic factors among women diagnosed with breast cancer aged 50-64 years. Cancer Epidemiol Biomarkers Prev 12: 1175-1181

Davis RP, Nora PF, Kooy RG, Hines JR (1979) Experience with lobular carcinoma of the breast. Emphasis on recent aspects of management. Arch Surg 114: $485-488$

Desai SB, Moonim MT, Gill AK, Punia RS, Naresh KN, Chinoy RF (2000) Hormone receptor status of breast cancer in India: a study of 798 tumours. Breast 9: 267-270

Dixon JM, Anderson TJ, Page DL, Lee D, Duffy SW (1982) Infiltrating lobular carcinoma of the breast. Histopathology 6: 149-161

Korhonen T, Huhtala H, Holli K (2004) A comparison of the biological and clinical features of invasive lobular and ductal carcinomas of the breast. Breast Cancer Res Treat 85: 23-29

Li CI, Anderson BO, Daling JR, Moe RE (2003a) Trends in incidence rates of invasive lobular and ductal breast carcinoma. JAMA 289: 1421-1424

Li CI, Malone KE, Porter PL, Weiss NS, Tang MT, Cushing-Haugen KL, Daling JR (2003b) Relationship between long durations and different regimens of hormone therapy and risk of breast cancer. JAMA 289: $3254-3263$

Li CI, Moe RE, Daling JR (2003c) Risk of mortality by histologic type of breast cancer among women aged 50 to 79 years. Arch Intern Med 163: $2149-2153$ lymph node status observed here may reflect differences in the utility of screening approaches to detect different histologic types of cancer. For example, it is well known that lobular tumours are more difficult to detect with mammography compared to ductal tumours, and this is thought to be primarily due to the fact that lobular tumours tend to grow as linear strands or sheets of cancer cells rather than as more discrete masses, explaining why they are more likely to be diagnosed at a more advanced stage (Davis et al, 1979; Dixon et al, 1982; Silverstein et al, 1994; Yeatman et al, 1995). Thus, currently, available breast cancer screening tools appear to be relatively less or relatively more effective in detecting different histopathologic types of breast cancer.

\section{ACKNOWLEDGEMENTS}

This study was supported by the National Cancer Institute (NCI), through a contract with the Fred Hutchinson Cancer Research Center and New Mexico State University (U56 CA096288).
Li CI, Weiss NS, Stanford JL, Daling JR (2000) Hormone replacement therapy in relation to risk of lobular and ductal breast carcinoma in middle-aged women. Cancer 88: 2570-2577

Mathieu MC, Rouzier R, Llombart-Cussac A, Sideris L, Koscielny S, Travagli JP, Contesso G, Delaloge S, Spielmann M (2004) The poor responsiveness of infiltrating lobular breast carcinomas to neoadjuvant chemotherapy can be explained by their biological profile. Eur J Cancer 40: $342-351$

Morabia A, Flandre P (1992) Misclassification bias related to definition of menopausal status in case-control studies of breast cancer. Int $J$ Epidemiol 21: 222-228

Newcomb PA, Titus-Ernstoff L, Egan KM, Trentham-Dietz A, Baron JA, Storer BE, Willett WC, Stampfer MJ (2002) Postmenopausal estrogen and progestin use in relation to breast cancer risk. Cancer Epidemiol Biomarkers Prev 11: 593-600

Newcomer LM, Newcomb PA, Potter JD, Yasui Y, Trentham-Dietz A, Storer BE, Longnecker MP, Baron JA, Daling JR (2003) Postmenopausal hormone therapy and risk of breast cancer by histologic type (United States). Cancer Causes Control 14: 225-233

Silverstein MJ, Lewinsky BS, Waisman JR, Gierson ED, Colburn WJ, Senofsky GM, Gamagami P (1994) Infiltrating lobular carcinoma. Is it different from infiltrating duct carcinoma? Cancer 73: 1673-1677

Stalsberg H, Thomas DB (1993) Age distribution of histologic types of breast carcinoma. Int J Cancer 54: 1-7

Stierer M, Rosen H, Weber R, Hanak H, Spona J, Tuchler H (1993) Immunohistochemical and biochemical measurement of estrogen and progesterone receptors in primary breast cancer. Correlation of histopathology and prognostic factors. Ann Surg 218: 13-21

Surveillance Epidemiology and End Results, National Cancer Institute (2005a) SEER data quality, available at http://seer.cancer.gov/about/ quality.html (accessed April 27, 2005)

Surveillance Epidemiology and End Results, National Cancer Institute (2005b) Characteristics of the SEER population compared with the total United States population, available at http://www.seer.cancer.gov/ registries/characteristics.html (accessed April 27, 2005)

Yeatman TJ, Cantor AB, Smith TJ, Smith SK, Reintgen DS, Miller MS, Ku NN, Baekey PA, Cox CE (1995) Tumor biology of infiltrating lobular carcinoma. Implications for management. Ann Surg 222: 549-559

Young Jr JL, Percy CL, Asire AJ (1981) Surveillance, epidemiology, and end results: incidence and mortality, 1973-77. Natl Cancer Inst Monogr 57: $1-1082$ 